\title{
Die Einwirkung von Acetylen auf die Oxyde des Kupfers.
}

Von

\author{
F. A. Gooch und De Forest Batdwin. ${ }^{1}$
}

In einer kürzlich erschienenen Mitteilung von ERDManN und KÖTHNER ${ }^{2}$ wurde die Bildung einer eigentümlichen hellbraunen, sehr voluminösen Substanz, die bei der Einwirkung von Acetylen unterhalb $250^{\circ}$ C. auf Kuprooxyd oder sogar - wenn auch langsamer auf Kupfer entsteht, beschrieben. Beim Überleiten von Acetylen über $1 \mathrm{~g}$ Kuprooxyd (dargestellt aus Kupfersulfat, Traubenzucker und Natriumhydroxyd) während 18 Stunden bildeten sich $7 \mathrm{~g}$ dieses Produktes, die ein Volumen von $300 \mathrm{ccm}$ einnahmen. Bei höherer Temperatur erhält man eine schwarze, kohlige Masse und bei Rotglut $\left(400-500^{\circ} \mathrm{C}\right)$ setzt sich Kohle in Form von Graphit ab. Das hellbraune, flockige Material gab mit Salzsäure Kuprochlorid; beim Destillieren mit Zinkstaub bildete sich ein Destillat, welches die Eigenschaften von Naphtenen zeigte, und bei höherer Temperatur und beim schnellen Erhitzen entstanden aromatische Verbindungen, unter denen sich Naphtalin und ein Kresol nachweisen liels. ERDMANN und KÖTHNER sprechen diesen Körper als ein sehr kompliziertes, aber nicht explosibles Acetylenkupfer an und stellen nach der Analyse für ihn die Formel $\mathrm{C}_{44} \mathrm{H}_{64} \mathrm{Cu}_{3}$ auf. Abgesehen von ihrer ganz ungewöhnlichen Zusammensetzung ist diese Formel besonders dadurch auffallend, dafs sie zum Ausdruck bringt, dals bei der Einwirkung von Acetylen auf Kuprooxyd ein Austritt von Kohlenstoff - nicht von Wasserstoff - stattfindet; diese Thatsache wäre aufserordentlich bemerkenswert im Lichte der Campbell'schen Versuche, ${ }^{3}$ nach denen sich beim Üborleiten von Acetylen über mit Palladium überzogenes Kupferoxyd bei $225-230^{\circ}$ Wasser bildet, während erst bei $315-320^{\circ}$ Kohlensäure unter Abscheidung eines schwarzen Körpers auftritt. Bei der sorgfältigen Prüfung der von ERdMans und KöthNER angegebenen Zahlen zeigte es sich jedoch, dafs die ange-

${ }^{1}$ Ins Deutsche übertragen von J. KoppeL.

${ }^{2} Z$. anorg. Chem. 18, 49.

${ }^{3}$ Amer. Chem. Journ. 17, 690. 
gebene Formel auf einem Irrtum bei der Berechnung beruht: das Verhältnis der Anzahl der Kohlenstoffatome zu den Wasserstoffatomen ist thatsächlich nach den angegebenen Daten 6.45:5.70, d. h. also, das neue Produkt enthält, wie auch zu erwarten war, im Vergleich zu Acetylen weniger Wasserstoff als dieses.

Bezüglich des Kupfergehaltes der neuen Substanz sind die analytischen Daten leider nicht eindeutig; denn die für "Kupferoxyd" gefundenen Gewichtszahlen sind ohne weitere Reduktion in Prozente „Kupfer" verwandelt. Wenn hier in den analytischen Daten ein Druckfehler vorliegen sollte, und die Prozente Kupfer korrekt sind, so betrüge der mittlere Prozentgehalt der Substanz an Kupfer 15.43; sind andererseits die analytischen Daten richtig und liegt der Fehler in der Berechnung, so betrüge der Kupfergehalt $12.92 \%$. Im ersteren Falle ergiebt sich beim Addieren der Analyse ein Manko von ca. $1.5 \%$ und in zweiten Falle von ca. $4 \%$, welches den thatsächlichen Gehalt der Substanz an Sauerstoff darstellen könne. Bei dieser Sachlage mufs die Existenz des Acetylenkupfers von Erdmann und KöTHNER zunächst in Zweifel gezogen werden.

Vor mehr als 30 Jahren wies BERTHELOT ${ }^{1}$ nach, dals Acetylen durch Hitze polymerisiert oder z. T. in Kohlenstoff und Wasserstoff zerlegt wird; diese Reaktionen finden leichter und bei niedrigeren Temperaturen statt, wenn metallisches Eisen zugegen ist; dabei bilden sich dann Kohle, Wasserstoff und aufserdem Verbindungen, welche von den durch Hitze allein gebildeten verschieden sind.

Moissan und Moureu ${ }^{2}$ beobachteten das Glühen von Acetylen beim Überleiten desselben über fein verteiltes Eisen, Kobalt, Nickel oder Platin bei gewöhnlicher Temperatur unter Bildung von Kohle, Wasserstoff und pyrogenen Verbindungen; sie fanden die Ursache dieses Verhaltens in dero Porsität der angewendeten Metalle.

Es würde indessen sehr natürlich sein, wenn die Gegenwart von Sauerstoff - frei oder gebunden - eine wichtige Rolle bei diesen Erscheinungen spielte, wie es auch bei der seltsamen, von GRUNER $^{3}$ beobachteten Reaktion zwischen Kohlenoxyd und mit Wasserstoff reduziertem Eisen der Fall zu sein scheint; dieser letztere Körper kann nur mit der allergröfsten Vorsicht in reinem Zustande dargestellt werden, wie MoIssan ${ }^{1}$ nachgewiesen hat, im allgemeinen enthält er beträchtliche Mengen von Ferrooxyd. Die Thatsache,

1 Ann. Chim. Phys. [4] 9, 448.

2 Compt. rend. 122, 1240.

s Ann. Ohim. Phys. [4] 26, 5. 
dal's das „Acetylenkupfer“" von ERDMANN und KötHNER viel leichter bei der Einwirkung von Kuprooxyd auf Acetylen entsteht, als bei der Reaktion zwischen Acetylen und metallischem Kupfer, legt die Vermutung nahe, dafs die oxydierende Wirkung des Kuprooxyds dieser Substanz ihre eigentümliche Aktivität erteilt. Es drängt sich dabei die Frage auf, ob das Kupfer in Wirklichkeit ein wesentlicher Bestandteil der Verbindung von ERDMaNN und KöThNER ist.

Bei unseren Versuchen über die Einwirkung von Acetylen auf die Oxyde des Kupfers (und anderer Elemente) leiteten wir das Gas (welches in der üblichen Weise durch die Einwirkung von Wasser auf Calciumkarbid hergestellt war und über Wasser aufbewahrt wurde) über das Oxyd, das sich in einem Porzellanschiffchen in einer Glasröhre von $50 \mathrm{~cm}$ Länge und $2 \mathrm{~cm}$ Durchmesser befand; die letztere wurde in einem kleinen Verbrennungsofen erhitzt. An seinen beiden Fnden war das Glasrohr mit Gummistopfen verschlossen, von denen der eine ein kleines Röhrchen zum Eintritt des Gases und ein Thermometer für hohe Temperaturen trug, welches so eingesetzt war, dafs seine Kugel sich gerade über dem Schiffchen mit Oxyd befand; in dem zweiten Gummistopfen war ein Wasserverschlufs eingesetzt. Bei den Vorversuchen wurde das Acetylen, wenn es über Wasser aufgefangen war, nicht weiter gereinigt; auch wurde es, da Wasser ein Reaktionsprodukt von Acetylen und Oxyden ist, nicht getrocknet; bei den späteren Versuchen wurde das Gas sorgfältig getrocknet und gereinigt, um analysenreine Substanz zu gewinnen.

Wir fanden, dafs sich der voluminöse Körper, der nach ERDMANN und KöTHNER bei der Einwirkung von Acetylen auf Kuprooxyd entsteht, am besten bei $225^{\circ}$ bildet. Bei dieser Temperatur füllt sich die Röhre bald mit einem flockigen Produkt und es bildet sich Wasser, doch trat keine merkliche Menge von Kohlendioxyd auf, wie auch $\mathrm{C}_{A M P B E L I}$ bei seinen Versuchen mit palladiniertem Kupferoxyd fand. Der Kupfergehalt des Produktes ist in der Probe und bei den verschiedenen Versuchen verschieden; wir fanden von 1.54-24.21\% der für die Verbrennung verwendeten Substanz.

Es scheint auch, als ob die Einwirkung von Acetylen auf Kuprioxyd fast genau so verläuft, wie die Einwirkung auf Kuprooxyd, nur dafs bei dem ersteren beim Beginn der Reaktion offenbar eine Reduktion eintritt. Die Kupfermenge in diesem Reaktionsprodukt schwankte bei unseren Versuchen von 6.53-21.30\%. Bei einem

1 Ann. Chim. Phys. [5] 21, 199. 
Versuche wurde ein Produkt, welches 9.34\% Kupfer enthielt, abermals der Einwirkung von Acetylen ausgesetzt, wobei sich wieder eine neue Menge der Substanz bildete, die nunmehr 3.87\% Kupfer enthielt.

Bei der Einwirkung von Acetylen auf eine sorgfältig im Wasserstoffstrom reduzierte und dann an einem Ende in der äufseren Bunsenflamme oxydierte Kupferspirale bei $225^{\circ}$ bildete sich das charakteristische Produkt nur an dem oxydierten Ende, während das nichtoxydierte Ende nur seine Farbe veränderte.

Während also metallisches Kupfer bei verhältnismäfsig hoher Temperatur die Polymerisation des Acetylens verursachen kann, zeigen diese Versuche, dafs die Bildung der eigentümlichen, in Rede stehenden Produkte auf eine bereits bei verhältnismälsig niedrigen Temperaturen beginnende Oxydationswirkung zurückzuführen ist. So fanden wir, dals Ferrioxyd beim Erhitzen in Acetylen auf 150 bis $360^{\circ}$ je nach den Umständen dunkel wird, erglüht und unter Entwickelung ron Wärme die Abscheidung eines dunklen, kohligen Produktes veranlafst. Die bei derartigen Reaktionen gebildeten Substanzen enthielten von $2.80-5.86 \%$ Eisen.

Silberoxyd wirkt gleichfalls auf Acetylen ein; bei einem Versuch trat bereits bei gewöhnlicher Temperatur die Reaktion ein, während, schon bevor die Temperatur auf $100^{\circ}$ gestiegen war, eine heftige Explosion stattfand, die das Schiffchen zertrümmerte und das Silber gegen die Wände der Glasröhre schleuderte.

Durch diese lokal heftige Explosion bei dem letzten Versuch war der Beweis geliefert, dals sich im ersten Stadium der Reaktion ein Acetylid bildete, welches sich später, wenn die Dissoziationstemperatur erreicht ist, zersetzt. Bei den Versuchen mit den Oxyden des Kupfers und Eisens liegt nun offenbar die Temperatur, bei welcher die Einwirkung des Acetylens beginnt, oberhalb der Temperatur, bei welcher sich die empfindlichen Acetylide dissoziieren würden, und wir haben deswegen in den beobachteten Erscheinungen keinen Beweis für die Bildung derartiger Verbindungen mit Kupfer und Eisen unter den angegebenen Versuchsbedingungen.

Unter Nr. 1 bis 3 der folgenden Tabelle sind die Versuchsresultate angegeben, die bei der Analyse verschiedener, durch Überleiten von Acetylen ${ }^{1}$ über Kuprooxyd erhaltener Produkte sich ergeben. Die Temperatur bei diesen Versuchen war $225^{\circ}$; im Laufe

${ }^{1}$ Zur Reinigung wurde das Acetylen durch eine salzsaure Merkurichloridlösung geleitet; es wurde über Kali getrocknet. 
einer halben Stunde war das Rohr vollkommen mit einer durch den Druck zusammengeprelsten Substanz angefüllt, die eine schwammige Masse von lichtbrauner Farbe an den äufseren Wänden der Röhre (1) darstellte, während sie im Schiffchen (2) dunkler war und am Boden des Schiffchens (3), wo ursprünglich das Kupferoxyd lag, eine fast schwarze Färbung zeigte.

Die unter Nr. 4 und 5 aufgeführten Analysen sind an Produkten ausgeführt, welche sich bei der Einwirkung von nicht besonders gereinigtem Acetylen auf Kuprioxyd gebildet hatten.

\begin{tabular}{|c|c|c|c|c|c|c|c|c|}
\hline \multirow{3}{*}{ 安 } & \multirow{3}{*}{$\begin{array}{c}\text { Angew. } \\
\text { Substanz } \\
\mathrm{g}\end{array}$} & \multicolumn{3}{|c|}{ Gefunden: } & \multicolumn{4}{|c|}{ Berechnet: } \\
\hline & & $\mathrm{CO}_{2}$ & $\overrightarrow{\mathrm{H}}_{2} \mathrm{O}$ & $\overline{\mathrm{CuO}}$ & $\overline{\mathrm{C}}$ & $\overline{\mathbf{H}}$ & $\mathrm{Cu}$ & $O$ (Diff) \\
\hline & & $g$ & $\mathrm{~g}$ & $g$ & $g$ & $\mathrm{~g}$ & $\mathrm{~g}$ & $\mathrm{~g}$ \\
\hline 1 & 0.1170 & 0.3978 & 0.0673 & 0.0022 & 0.1085 & 0.0075 & 0.0018 & -0.0008 \\
\hline 2 & 0.2247 & 0.7489 & 0.0979 & - & 0.2042 & 0.0109 & - & - \\
\hline $\mathbf{3}$ & 0.1096 & 0.3678 & 0.0488 & 0.0045 & 0.1003 & $0.004 \overline{5}$ & 0.0036 & 0.0003 \\
\hline 4 & 0.1360 & 0.4116 & 0.0579 & 0.0182 & 0.1123 & 0.0064 & 0.0146 & 00027 \\
\hline \multirow[t]{7}{*}{5} & 0.1188 & 0.3098 & 0.0461 & 0.0317 & 0.0845 & 0.0051 & 0.0253 & 0.0039 \\
\hline & & & 1 & 2 & 3 & \multicolumn{2}{|c|}{4} & 5 \\
\hline & \multirow{4}{*}{\multicolumn{2}{|c|}{$\begin{array}{l}\text { Kohlenstoff } \\
\text { Wasserstoff } \\
\text { Kupfer } \\
\text { Sauerstoff }\end{array}$}} & 92.74 & 90.88 & 91.51 & \multicolumn{2}{|c|}{82.57} & $71.13 \%$ \\
\hline & & & 6.41 & 4.85 & 4.95 & \multicolumn{2}{|c|}{4.71} & 4.29, \\
\hline & & & 1.54 & - & 3.29 & \multicolumn{2}{|c|}{10.74} & $21.30 "$ \\
\hline & & & - & - & 0.27 & \multicolumn{2}{|c|}{1.98} & 3.28, \\
\hline & & & 100.69 & & $100.0 \mathrm{C}$ & \multicolumn{2}{|c|}{100.00} & $100.00 \%$ \\
\hline
\end{tabular}

Der in diesen Produkten vorhandene Sauerstoff ist offenbar dem Kupfergehalte proportional und seine Menge ist niemals gröfser, als sich unter der Annahme berechnen lielse, dals etwas von dem ursprünglich vorhandenen Oxyd seinen Sauerstoff noch enthält. So weit sich aus den Analysen erkennen lärst, enthält die Substanz von der hellsten Farbe (1) sehr wenig Kupfer und keinen Sauerstoff; das dunkelste Produkt (3), welches aus dem Kuprooxyd gebildet wurde, enthält eine Sauerstoffmenge, die einem Gemisch von 2 Teilen Kupfer mit 3 Teilen Kuprooxyd entspricht; die Sauerstoffmengen von Produkt (4) und (5), welche bei der Einwirkung von Acetylen auf Kuprioxyd erhalten wurden, entsprechen annähernd einem Gemisch von Kupro- und Kuprioxyd zu gleichen Teilen.

Diese .Thatsache - in Verbindung mit der grofsen Veränderlichkeit des Kupfergehaltes und dem Umstande, dafs gerade das Produkt, welches am wenigsten durch mechanisch eingeschlossenes 
Metall oder Oxyd verunreinigt werden kann, auch das Minimum an Kupfer enthält - legt den Gedanken nahe, dafs der vorhandene Sauerstoff höchstwahrscheinlich an Kupfer gebunden ist und dals Kupfer mechanisch als Metall oder Oxyd includiert wird, dafs es aber kein wesentlicher Bestandteil der organischen Verbindung ist. Beachtet man also das Kupfer und die Kupferoxyde nicht weiter, und berechnet die Zusammensetzung der entstandenen Produkte unter der Annahme, dafs sie im wesentlichen aus Kohlenstoff und Wasserstoff bestehen, so kommt man zu folgender Zusammenstellung:

\begin{tabular}{|c|c|c|c|c|c|}
\hline & 1 & 2 & 3 & 4 & 5 \\
\hline Kohlenstoff & 93.54 & 94.93 & 94.88 & 94.60 & $94.31 \%$ \\
\hline \multirow[t]{2}{*}{ Wasserstoff } & 6.46 & 5.07 & 5.12 & 5.40 & 5.69, \\
\hline & 100.00 & 100.00 & 100.00 & 100.00 & $100.00 \%$ \\
\hline
\end{tabular}

Diese Zahlen entsprechen Formeln von $\mathrm{C}_{12} \mathrm{H}_{10}$ bis $\mathrm{C}_{16} \mathrm{H}_{10}$, im Durchschnitt also $\mathrm{C}_{14} \mathrm{H}_{10}$, der Formel für Anthracen oder Paraanthracen. Die analytischen Daten von ERDManN und KöTHNER lassen im Durchschnitt auf ein Produkt schliefsen, dessen Zusammensetzung am besten auf die erste der oben angegebenen Formeln parst und weniger gut mit den übrigen übereinstimmt. Das entstehende Produkt ist zweifellos je nach Temperatur und nach Oxydationswirkung verschieden. So begann bei einem Versuch, bei dem Acetylen über Ferrioxyd geleitet wurde, die Reaktion bei $365^{\circ}$ unter Erglühen, wie bereits von Morssan und Moureu ${ }^{1}$ beschrieben ist, und die Analyse der gebildeten Substanz $(\mathrm{C}=91.53, \mathrm{H}=1.36, \mathrm{Fe}=5.85, \mathrm{O}=1.26)$ zeigt ein Verhältnis von Kohle zu Wasserstoff, welches viermal so grofs ist, als es durchschnittlich bei den bei $225^{\circ}$ aus den Kupferoxyden entstandenen Körpern gefunden wurde.

Als Resultat der vorstehenden Untersuchung ergiebt sich also der Schlufs, dafs kein Beweis dafür vorhanden ist, dafs das bei der Einwirkung von Acetylen auf die Oxyde des Kupfers unter den geschilderten Versuchsbedingungen entstehende Produkt etwas anderes als ein Gemisch eines oder mehrerer Kohlenwasserstoffe mit metallischem Kupfer oder mit Kupferoxyden ist, welches in seinen dunkleren Teilen wahrscheinlich noch freien Kohlenstoff enthält.

1 l. c.

New Haven, U. S. A., The Kent Chemical Laboratory of Yale University. Bei der Redaktion eingegangen am 13. August 1899. 